\title{
Composites
}

\section{An alternative synthesis route to graphene oxide: influence of surface chemistry on charge transport in epoxy-based composites}

\author{
Orestis Vryonis $^{1, *}$ (D), Thomas Andritsch ${ }^{1}$ (D), Alun S. Vaughan ${ }^{1}$ (D), and Paul L. Lewin ${ }^{1}$ (D) \\ ${ }^{1}$ Tony Davies High Voltage Laboratory, Department of Electronics and Computer Science, Faculty of Engineering and Physical \\ Sciences, University of Southampton, Southampton, UK
}

Received: 26 November 2018

Accepted: 21 February 2019

Published online:

27 February 2019

(C) The Author(s) 2019

\begin{abstract}
A synthetic route for the production of graphene oxide is described, in which the commonly used potassium permanganate $\left(\mathrm{KMnO}_{4}\right)$ is replaced by chromium trioxide $\left(\mathrm{CrO}_{3}\right)$ as the oxidizing agent. Raman spectroscopy, thermogravimetric analysis and X-ray photoelectron spectroscopy demonstrate that the product is characterized by a reduced level of oxidation and a reduced defect content, compared to conventional graphene oxide (GO). We therefore term the product moderately oxidized graphene oxide (mGO). In comparison with $\mathrm{GO}$, it is shown that when introduced into an epoxy matrix, mGO offers significant potential benefits. These include: excellent compatibility with the epoxy matrix leading to a low percolation threshold for electrical conductivity ( $\sim 0.5 \mathrm{vol} \%)$; an associated increase in electrical conductivity of about eight orders of magnitude; no adverse influence on the epoxy curing reactions; potentially simplified material processing strategies.
\end{abstract}

\section{Introduction}

Graphite is a natural layered material, the crystal lattice of which consists of an assembly of two-dimensional $s p^{2}$ hybridized graphene sheets stacked together. A key prerequisite for the exploitation of graphite's properties as a polymer filler [1] is, therefore, layer separation and dispersion within the host matrix. One strategy for this led to so-called expanded graphite (EG), which is commonly produced by acidic intercalation of graphite, followed by exposure to elevated temperatures and thermal shock [2]. The isolation of graphene in 2004 [3] has subsequently generated vast global interest regarding its extraordinary properties $[4,5]$, and the technological utilization of graphene nanoplatelets (GNP) has, over the last decade or so, been extensively explored [6, 7]. These layered structures can be produced by several routes, as reviewed by Jang et al. [8], which usually include a further step after the production of EG in order to reduce the size to the nanometric scale. An alternative route to layer exfoliation is through acidic

Address correspondence to E-mail: O.Vryonis@soton.ac.uk 
oxidation and synthesis of oxidized graphite, consisting of graphene oxide (GO) layers [9]. GO exhibits a layered structure in which the surfaces are decorated with a range of oxygen-based functionalities, resulting in a mixed configuration composed of both $s p^{2}$ and $s p^{3}$ hybridized carbon atoms [10]. Despite debate concerning the precise chemical structure of graphene oxide, the most widely adopted configuration (Lerf-Klinowski model) [11] involves basal planes decorated with epoxide and hydroxyl groups, while the peripheral planes contain carbonyl and carboxyl groups. The existence of these groups, which can be considered as defects within an ideal graphite layer, adversely affects the intrinsic electrical $[12,13]$ and thermal [14] properties of the material, while beneficially increasing hydrophilicity [10], inter-layer distance [15], organic solvent solubility [16] and compatibility with polymer matrices [17]. All of these latter factors enhance dispersion within polar solvents/polymers [18, 19] and can ease the production of polymer-based nanocomposites.

While many methods for the exfoliation/disaggregation of graphite have been described, these can broadly be divided into five main categories [20]. The basic principle of these processes involves the intercalation of graphite in an acidic medium, combined with reaction with an oxidant. The earliest recorded method was reported by Brodie [21], utilizing potassium chlorate $\left(\mathrm{KClO}_{3}\right)$ as an oxidant and fuming nitric acid $\left(\mathrm{HNO}_{3}\right)$ as the reaction medium. Later, the reaction medium was modified, first by Staudenmaier [22] by the addition of sulphuric acid $\left(\mathrm{H}_{2} \mathrm{SO}_{4}\right)$ and then by Hofmann [23] by the usage of nonfuming $\mathrm{HNO}_{3}$. All of these methods were hazardous and protracted (about 4 days). The mostly commonly used method was developed by Hummers [24] and involves the use of potassium permanganate $\left(\mathrm{KMnO}_{4}\right)$ and sodium nitrate $\left(\mathrm{NaNO}_{3}\right)$ as oxidants in sulphuric acid $\left(\mathrm{H}_{2} \mathrm{SO}_{4}\right)$ and, in this way, the duration of the process was reduced to just a few hours. Recently, it was shown that omission of the highly toxic $\mathrm{NaNO}_{3}$ results in the same quality of product [25]. Finally, further refinement was reported by Tour [26] with the usage of only $\mathrm{KMnO}_{4}$ as oxidant and a mixture of $\mathrm{H}_{2} \mathrm{SO}_{4}$ and phosphoric acid $\left(\mathrm{H}_{3} \mathrm{PO}_{4}\right)$ as the acidic reaction medium. The final two methods are the safest, since they exclude the usage of $\mathrm{KClO}_{3}$, which can be explosive, $\mathrm{HNO}_{3}$, which can produce acidic fog, and $\mathrm{NaNO}_{3}$.
Since $\mathrm{KMnO}_{4}$ in acidic solution produces highly oxidizing species $[9,25]$, GO produced using associated methods are characterized by high oxygen contents [25, 26]; this equates to a high degree of structural disruption, which can have adverse consequences for the physical properties of the system. Also, if directly incorporated into epoxy resins at significant loading levels, crosslinking of the system can be disrupted [27, 28], resulting in reduced glass transition temperature $\left(T_{\mathrm{g}}\right)$ of the matrix polymer. The associated reactions between GO and epoxy were investigated and presented elsewhere [29]. Therefore, GO produced in this way is either used sparingly to modify mechanical performance [30] or further processed by reduction [31, 32] or functionalization [33-35]. In some cases, even the functionalization of GO was not sufficient to prevent alteration of the epoxy stoichiometry [36, 37]. Where high filler loading levels are required in order to form percolating structures, for example, to enhance electrical or thermal conduction, the direct usage of GO in epoxy nanocomposites is problematical. Consequently, in order to avoid such phenomena, EG/GNP or reduced GO (rGO), should be ideally used, which exhibit reduced defect contents and do not disrupt the epoxy crosslinking. Alternative methods such as electrochemical exfoliation [38-40] could be used, in order to tailor the oxygen content. The downside (apart from poor compatibility for the two former systems) is that the production of such systems involves multistep processing/synthesizing routes, which both adds complexity and reduces yields.

The study reported here was undertaken to explore the effectiveness of graphite oxidation by chromium in place of manganese. Chromium trioxide $\left(\mathrm{CrO}_{3}\right)$ is known as an effective intercalant [41, 42] but has previously been reported only weakly to react with graphite [43]. Therefore, by using $\mathrm{CrO}_{3}$, we sought to reduce the extent of graphite oxidation, resulting in a moderately oxidized graphene oxide (mGO), in order: (a) to reduce the defect content of the GO layers; (b) to minimize perturbation of the epoxy matrix crosslinking reactions; (c) to maintain sufficient compatibility with the matrix polymer. 


\section{Experimental methods}

\section{Materials}

Chromium (VI) trioxide was purchased from Fischer scientific (11390939, supplied by Alfa Aesar $^{\mathrm{TM}}$ ), $\mathrm{KMnO}_{4}$ was purchased from AnalaR (26910 VWR International) and $\mathrm{H}_{2} \mathrm{SO}_{4}$ from Sigma-Aldrich (79620). The precursor graphite and the hydrogen peroxide $\left(\mathrm{H}_{2} \mathrm{O}_{2}\right)$ solution (35\%) were also supplied by Sigma-Aldrich (496596 and 349887, respectively). The epoxy resin used was an amine-cured, bisphenol A-based system, namely Epilox ${ }^{\mathrm{TM}}$ Infusion System 5300 (LEUNA-Harze GmbH). This resin was chosen for this study because its low viscosity ( $\sim 275 \mathrm{mPas}$ / $25{ }^{\circ} \mathrm{C}$ ) makes it appropriate for structural fibre-reinforced composites in wind energy applications, the application of interest. The two parts used are ER 5300 as epoxy monomer (epoxy equivalent weight: $170 \mathrm{~g} / \mathrm{eq}$ ) and EC 5310 as hardener (amine value: $520 \mathrm{mg} \mathrm{KOH} / \mathrm{g}$ ) components, respectively.

\section{Synthesis of graphene oxide}

A modified Tour method was used to synthesize GO, to provide a comparator system for the chromiumsynthesized mGO. For this, $40 \mathrm{ml}$ of a $\mathrm{H}_{2} \mathrm{SO}_{4} / \mathrm{H}_{3} \mathrm{PO}_{4}$ mixture (5:2 per volume) was mixed with $1.8 \mathrm{~g}$ of $\mathrm{KMnO}_{4}$ and stirred at $50{ }^{\circ} \mathrm{C}$ to give a homogeneous solution. Afterwards, $300 \mathrm{mg}$ of graphite were added to the solution and left for $4 \mathrm{~h}$ under mild shaking. Another batch was prepared and left for $30 \mathrm{~min}$ to demonstrate the effect of the process duration. The system was neutralized by transferring into $100 \mathrm{ml}$ of cool distilled water, to which, $10 \mathrm{ml}$ of the $\mathrm{H}_{2} \mathrm{O}_{2}$ solution was added. Centrifugation (Heraeus ${ }^{\mathrm{TM}}$ Megafuge $^{\mathrm{TM}}$ 8) at $8872 \mathrm{~g}$ for $10 \mathrm{~min}$ was used to retrieve the GO and remove the supernatant acid. The sediment was re-dispersed and washed in distilled water (three times), ethanol (once) and acetone (twice); after each step, the GO was recovered from the suspension using centrifugation. A final GO/ acetone solution was obtained and probe sonicated (UP 200S) for $30 \mathrm{~min}$ with a 7-mm-diameter tip; the resulting GO could be incorporated into the epoxy only within this solvent, since subsequent drying led to a material that was too agglomerated to be dispersed. Henceforth, the resulting oxidized graphite will be referred to as GO.

\section{Synthesis of moderately oxidized graphene oxide}

For this synthesis, the graphite/acid ratio was the same as in the previous method, but now, only $\mathrm{H}_{2} \mathrm{SO}_{4}$ was used as the reaction medium. A saturated solution was produced by adding $\sim 20 \mathrm{mg}$ of $\mathrm{CrO}_{3}$ per $\mathrm{ml}$ of $\mathrm{H}_{2} \mathrm{SO}_{4}$ under continuous magnetic stirring at $50{ }^{\circ} \mathrm{C}$; to assist dissolution of the $\mathrm{CrO}_{3}$, the as-received crystals were first ground to a fine powder using a mortar and pestle. The resulting orangecoloured solution was poured into a glass flask containing the appropriate amount of graphite and was placed on a shaker for $30 \mathrm{~min}$ for the oxidation to proceed. After this time, the system was neutralized, centrifuged, washed and sonicated using the same conditions described above. Finally, the mGO was dried at $100{ }^{\circ} \mathrm{C}$ overnight since, in this case, this procedure did not lead to a highly agglomerated product. Visual comparison of suspensions in acetone of GO (yellowish suspension to the left in Fig. 1) and mGO (greyish suspension to the right in Fig. 1) reveals a marked difference in colour, which we interpret in terms of a shift in the band edge resulting from changes in the functional group density on the GO surface [44].

\section{Epoxy nanocomposite preparation}

Epoxy/mGO nanocomposites were prepared as follows: the epoxy was first mixed for 5 min with the

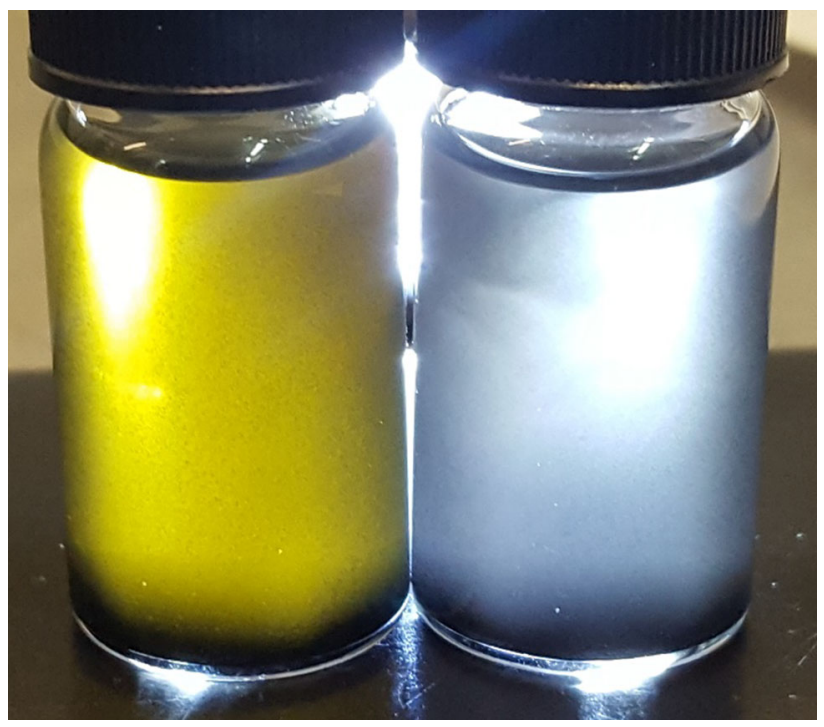

Figure 1 Suspensions in acetone of GO (yellowish suspension to the left) and $\mathrm{mGO}$ (greyish suspension to the right). 
appropriate amount of dry filler, using a planetary mixer (Speedmixer ${ }^{\mathrm{TM}}$ DAC $150.1 \mathrm{FV}$ ) operating at $3000 \mathrm{rpm}$. Although it is more normal initially to distribute GO in a solvent to aid dispersion, this was found to be unnecessary in the case of our mGO, which beneficially obviates the need for subsequent solvent removal. From a technological perspective, direct dispersion without the need for the involvement of additional processing steps may be advantageous [34]. Furthermore, in our experience, the use of solvent-based preparation methods in conjunction with low-viscosity epoxy resins, like the one used in this study, can increase the propensity for particle sedimentation [45] and deteriorated final material performance, particularly when the GO is lightly functionalized. Subsequently, the hardener was added in a stoichiometric weight ratio of $10 / 2$ (epoxy/hardener), as suggested by the supplier. The mixture was then vacuum degassed to remove entrained air and cast into moulds with the required dimensions. Curing was conducted at $70{ }^{\circ} \mathrm{C}$ for $6 \mathrm{~h}$, as suggested by the epoxy manufacturer.

\section{Characterization techniques}

The dry graphite, GO and mGO powders were characterized by Raman spectroscopy, in order to evaluate the structural alterations induced by the imposed oxidation process. For this, a Renishaw RM1000 confocal microprobe system was used, using a $780 \mathrm{~nm}$ excitation wavelength and operating at 2.5 $\mathrm{mW}$ power. A Perkin-Elmer Pyris 1 was used to assess the extent of the graphite oxidation via thermogravimetric analysis (TGA). Graphite, GO and mGO samples were first held at $70{ }^{\circ} \mathrm{C}$ for $10 \mathrm{~min}$ before being heating at $10{ }^{\circ} \mathrm{C} / \mathrm{min}$ to $700{ }^{\circ} \mathrm{C}$ in a nitrogen atmosphere. Elemental analysis of the examined powders was performed with a monochromated $\mathrm{Al} \mathrm{K} \alpha$ X-ray photoelectron spectroscopy (XPS) instrument (Kratos Axis Ultra DLD), with a typical resolution of $0.47 \mathrm{eV}$. The nanocomposite systems were morphologically characterized via scanning electron microscopy (SEM) using an EVO LS25, Zeiss instrument, and transmission electron microscopy (TEM) using an FEI Tecnai T12, instrument. For TEM, sections were prepared using a Leica Ultracut E ultramicrotome together with a Diatome $45^{\circ}$ diamond knife.

The glass transition temperature $\left(T_{\mathrm{g}}\right)$ of all the epoxy-based systems (unfilled resin and nanocomposites) was determined by differential scanning calorimetry (DSC), using a Perkin-Elmer DSC7. For this, $T_{\mathrm{g}}$ was evaluated from the point of inflection of the step-like transition of the heat flow while heating up to $120^{\circ} \mathrm{C}$ at a rate of $10{ }^{\circ} \mathrm{C} / \mathrm{min}$. Prior to this, the respective system's thermal history was erased [46] by heating and cooling over the same temperature range at the same scan rate. A total of three repeats were used to calculate average and standard deviation values for the $T_{\mathrm{g}}$ of each material system. DC electrical conductivity was determined by means of two-probe methods using two different sample thicknesses for repeatability purposes and to exclude possible geometric factors from influencing the derived values. The thick samples were disc-shaped, $3 \mathrm{~mm}$ in thickness, and their surfaces were polished with abrasive paper (grade 1000) and then goldcoated. An Agilent 34401A digital multimeter and guarded, disc-shaped, aluminium, sample holders were used; the applied voltage was $150 \mathrm{~V}$. The thin samples were rectangular shaped, $\sim 200 \mu \mathrm{m}$ in thickness and their surfaces were, again, polished with abrasive paper (grade 1000). A Keithley 6517B electrometer/high resistance metre fitted with an 8009-resistivity fixture was used with the applied voltage being $100 \mathrm{~V}$ for $6 \mathrm{~min}$. Both evaluation methods produced comparable results, indicating that conduction at the chosen voltage was Ohmic; the values quoted are the average of all measurements, together with the associated standard deviation. 
Table of Abbreviations

\begin{tabular}{llll}
\hline Abbreviation & Meaning & Abbreviation & Meaning \\
\hline $\mathrm{KMnO}_{4}$ & Potassium permanganate & $\mathrm{GNP}$ & Graphene nanoplatelets \\
$\mathrm{CrO}_{3}$ & Chromium trioxide & $\mathrm{KClO}_{3}$ & Potassium chlorate \\
$\mathrm{TGA}$ & Thermogravimetric analysis & $\mathrm{HNO}_{3}$ & Nitric acid \\
$\mathrm{XPS}$ & X-ray photoelectron spectroscopy & $\mathrm{H}_{2} \mathrm{SO}_{4}$ & Sulphuric acid \\
$\mathrm{mGO}$ & Moderately oxidized graphene oxide & $\mathrm{NaNO}_{3}$ & Sodium nitrate \\
$\mathrm{GO}$ & Graphene oxide & $\mathrm{H}_{3} \mathrm{PO}_{4}$ & Phosphoric acid \\
$\mathrm{EG}$ & Expanded graphite & $T_{\mathrm{g}}$ & Glass transition temperature \\
$\mathrm{rGO}$ & Reduced GO & $\mathrm{SEM}$ & Scanning electron microscopy \\
$\mathrm{H} \mathrm{O}_{2}$ & Hydrogen peroxide & $\mathrm{TEM}$ & Transmission electron microscopy \\
$\mathrm{DSC}$ & Differential scanning calorimetry & $\mathrm{I}_{(\mathrm{D})} /(\mathrm{G})$ & Raman intensity ratio \\
$W_{\mathrm{f}}$ & Weight fraction & $\mathrm{FWHM}$ & Full width at half maximum \\
$V_{\mathrm{f}}$ & Volume fraction & $\rho_{\mathrm{m}}$ & Matrix density \\
$\rho_{\mathrm{f}}$ & Filler density & $\sigma_{\mathrm{c}}$ & Electrical conductivity of the composite \\
$\sigma_{\mathrm{f}}$ & Electrical conductivity of the filler & $t$ & Critical exponent for electrical conductivity \\
$V_{\mathrm{c}}$ & Critical volume fraction & $P_{\mathrm{t}}$ & Percolation threshold \\
$\mathrm{fGO}$ & Functionalized graphene oxide & $\sigma_{\mathrm{m}}$ & Electrical conductivity of the unfilled matrix \\
$\sigma_{\mathrm{max}}$ & Maximum electrical conductivity reported & $\mathrm{UV} / \mathrm{O}_{3}$ & Ultraviolet/Ozone \\
$\mathrm{THF}$ & Tetrahydrofuran & $\mathrm{CHCl}$ & Chloroform \\
\hline
\end{tabular}

\section{Results}

\section{Effect of oxidant chemistry on graphite oxidation}

The research hypothesis that underpins the work reported here is that changing the oxidizing medium from a widely used manganese-based system to one based upon chromium leads to a change in the oxidation state of the oxidized graphite: specifically, the production of material that is characterized by a reduced degree of oxidation/disorder. This issue will therefore be addressed first, using three complementary approaches.

Raman spectra obtained from the graphite precursor, the two GO specimens and the $\mathrm{mGO}$ are presented in Fig. 2. A total of four characteristic bands can be observed at 1307, 1569, 1602 and $2610 \mathrm{~cm}^{-1}$, namely D, G, $\mathrm{D}^{\prime}$ and $2 \mathrm{D}$, respectively. The $\mathrm{G}$ band is associated with the first-order scattering of $\mathrm{E}_{2 \mathrm{~g}}$ phonons, representing the in-phase vibrations of the $s p^{2}$ carbon lattice $[47,48]$, while the $\mathrm{D}$ and $\mathrm{D}^{\prime}$ bands are associated with the extent of disorder $[49,50]$. The $2 \mathrm{D}$ band, also termed the $\mathrm{G}^{\prime}$ band, is the overtone of the $\mathrm{D}$ band and is associated with the highly ordered stacking sequence of crystalline graphite [50].

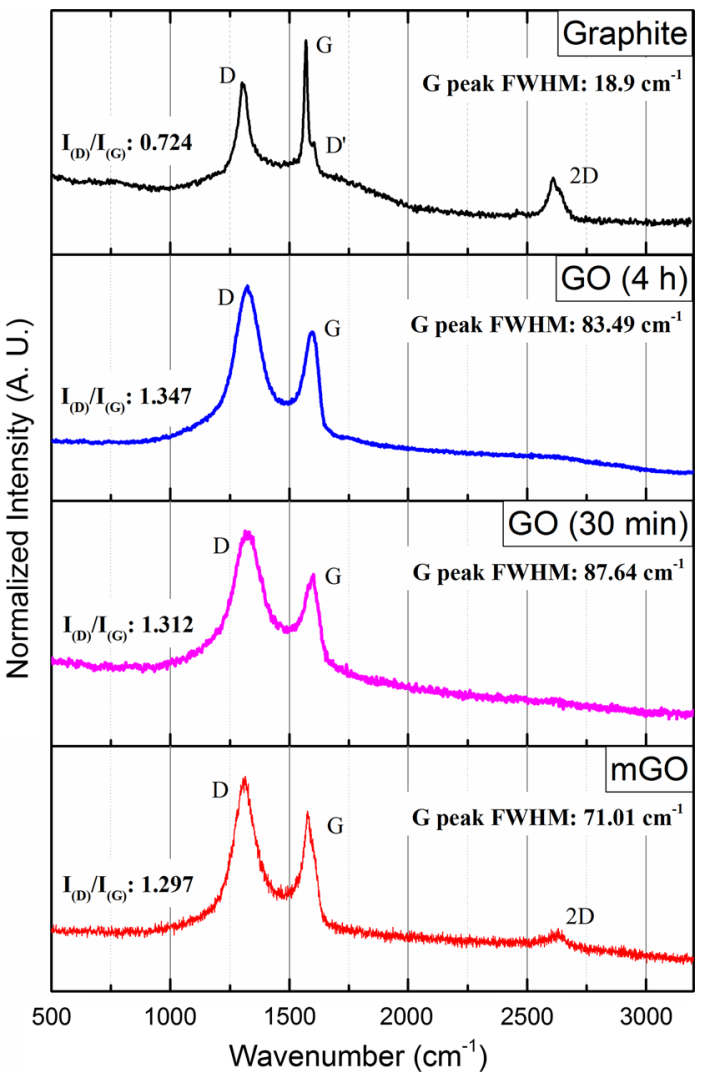

Figure 2 Raman spectra (excitation $780 \mathrm{~nm}$ ) of the precursor graphite (black), mGO (red) and GO obtained by $4 \mathrm{~h}$ (blue) and 30 min (pink) oxidation duration. 
The Raman response of the precursor graphite is dominated by vibrations of the carbon atoms (sharp and intense $G$ band) but also contains evidence of defects, as evinced by the presence of the $\mathrm{D}$ and $\mathrm{D}^{\prime}$ bands. In contrast, the GO Raman spectra reveal broad $G$ bands shifting slightly to higher wave numbers with increasing oxidation duration. The sample oxidized for $30 \mathrm{~min}$ displayed a $G$ peak at $1583 \mathrm{~cm}^{-1}$, while the respective peak for the sample oxidized for $4 \mathrm{~h}$ was located at $1586 \mathrm{~cm}^{-1}$. This indicates resonance at higher frequencies, which has been attributed to the presence of isolated double bonds compared to the graphite [47]. Reasonably, here, this phenomenon parallels changes in oxidation time while, elsewhere, it has been reported to occur as a result of increasing the amount of $\mathrm{KMnO}_{4}$ [51]. Both GO samples exhibit considerably broadened D bands, which are more pronounced than the respective $G$ bands. This is an indication of oxygen-based groups being attached on the graphitic lattice, decreasing the size of the in-plane $s p^{2}$ domains [47] through $s p^{3}$ bonding [51]. Also, the $2 \mathrm{D}$ band is not visible in either GO sample, which is reasonable given that the stacking order will be heavily disrupted by the inherent structural imperfections [51]. In the case of $\mathrm{mGO}$, the $\mathrm{G}$ band is located at 1576 $\mathrm{cm}^{-1}$, a lower wave number than in the GO samples but higher than graphite. Nevertheless, the G band is still broadened and less intense compared to the sample's D band and, in this respect, the mGO mirrors the Raman behaviour of the GO. This indicates that while the graphitic structure is disrupted, the extent of the disruption is reduced compared with the GO. The latter is also confirmed by the presence of the 2D band (albeit weak) in the mGO Raman spectrum, which suggests that some stacking order is preserved after the oxidation process, contrarily to both GO samples. The $I_{(\mathrm{D})} /$ (G) $_{\mathrm{G})}$ intensity ratios for the precursor graphite, GO (4 h oxidation), GO (30 min oxidation) and mGO were: $0.724,1.347,1.312,1.297$, respectively, as portrayed in Fig. 2. This ratio is commonly used as an indication of the induced disorder in the system, and the acquired results clearly align with this, showing smaller $I_{(\mathrm{D})} /$ (G) $_{\text {G }}$ values for the mGO compared to both GO samples. Nevertheless, discrepancies have been observed before [51], leading to the assumption that there is no straightforward relation between the intensity ratio and the oxidation level. Full width at half maximum (FWHM) of the observed G peaks (see Fig. 2) were 18.9, 83.49, 87.64,
$71.01 \mathrm{~cm}^{-1}$ for the precursor graphite, GO $(4 \mathrm{~h}$ oxidation), GO (30 min oxidation) and $\mathrm{mGO}$, respectively. This value has also been used [51] to reflect the oxidation level, by showing increasing dependency with it. It is apparent that the precursor graphite displays the smallest values among the examined samples. While a slight discrepancy was observed for the two GO samples, the mGO shows a considerably lower FWHM value compared to them.

TGA is commonly used to evaluate the concentration of oxygen groups attached to the graphitic structure, since this determines the weight loss at specific temperatures [52]. Figure 3 compares TGA data obtained from the precursor graphite, the $\mathrm{mGO}$ and the samples of GO prepared using the two different oxidation times. When heated up to $700{ }^{\circ} \mathrm{C}$, the graphite displays a minimal mass loss; the small observed reduction in mass is ascribed to the presence of impurities/defects and therefore aligns with the evidence of defects provided by the Raman data shown above. In contrast, all the systems that were subjected to oxidation (both GO and mGO) show three weight loss processes. The small mass loss that occurs up to $200{ }^{\circ} \mathrm{C}$ is ascribed to removal of adsorbed water [16, 25]. Between 200 and $300{ }^{\circ} \mathrm{C}$, the rate of mass loss is increased, while further heating results in a gradual, monotonic reduction in sample mass; these two processes have, respectively, been related to the removal of labile and stable oxygen-based groups [26]. In the case of both GO powders, removal of labile oxygen-based moieties leads to a mass

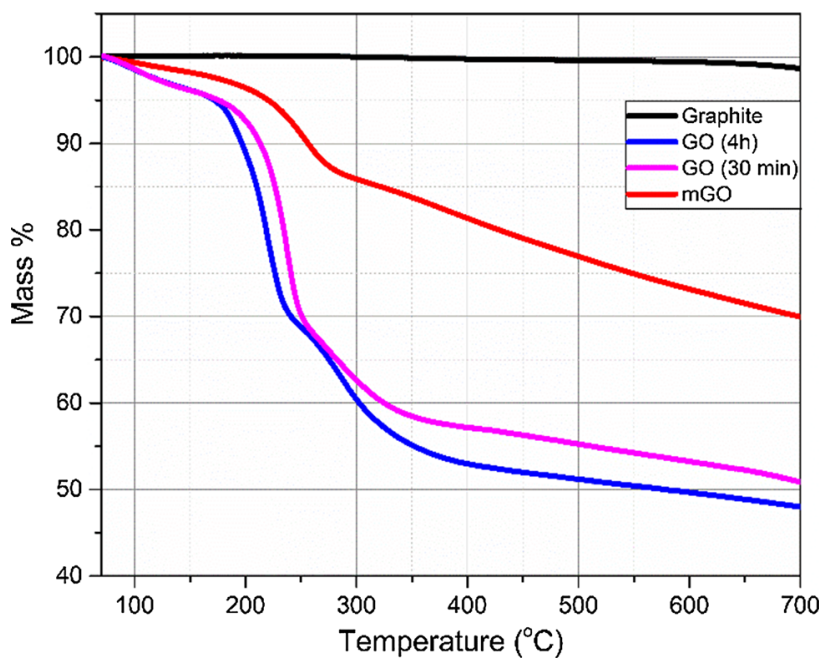

Figure 3 TGA plots for precursor graphite (black), mGO (red) and GO obtained by $4 \mathrm{~h}$ (blue) and $30 \mathrm{~min}$ (pink) oxidation duration. Heating rate $10{ }^{\circ} \mathrm{C} / \mathrm{min}$, nitrogen environment. 
reduction of more than $30 \%$, a figure that aligns well with published data $[25,26]$. Evidently, varying the oxidation time within the range considered here does not lead to very different changes in sample mass between 200 and $300{ }^{\circ} \mathrm{C}$, albeit that the mass loss does appear to be slightly larger in the case of the system oxidized for the longer time. This implies that the graphite surface chemistry is rapidly disrupted by the manganese-based oxidizing agent, since extending the duration of the process by eightfold results in only a minor change in the product. For this reason, only one oxidation time (30 $\mathrm{min})$ was used for the synthesis of the $\mathrm{mGO}$, since it leads to a saving in processing time and aligns with the objective to reduce the extent of oxidation. The TGA data shown above indicate that using $\mathrm{CrO}_{3}$ in place of $\mathrm{KMnO}_{4}$ leads to a significant reduction in mass loss $(\sim 10 \%)$ between $200{ }^{\circ} \mathrm{C}$ and $300{ }^{\circ} \mathrm{C}$, implying that the mGO structure contains proportionally fewer labile oxygen groups. Indeed, our directly synthesized mGO (i.e. without being subjected to any subsequent reduction process) displays a smaller related mass loss than either chemically reduced GO (via reaction with hydrazine hydrate) [53] or microwave reduced GO [32].

Figure 4 shows XPS data obtained from the precursor graphite, the GO and the mGO powders. Figure $4 \mathrm{a}$ shows the overall spectra, while Fig. $4 \mathrm{~b}$
Table 1 Percentage elemental contents calculated from the XPS analysis

\begin{tabular}{llcl}
\hline Sample & C (\%) & O (\%) & S (\%) \\
\hline Graphite & 96.8 & 3.2 & 0.00 \\
GO & 72.8 & 26.3 & 0.9 \\
mGO & 83.3 & 16.3 & 0.4 \\
\hline
\end{tabular}

presents the C1s peak at high resolution. Derived elemental ratios are presented in Table 1.

It can be seen that the precursor graphite contains some minor oxygen impurities, resulting in a $\mathrm{C} / \mathrm{O}$ ratio of $\sim 30$, which is consistent with the Raman data presented above. The synthesized GO shows a much lower $\mathrm{C} / \mathrm{O}$ ratio of $\sim 2.8$ and an oxygen content of $\sim 26 \%$. These values are typical for conventional GO, where $\mathrm{C} / \mathrm{O}$ ratio values between 2 and 4 have been reported [54]. The $\mathrm{C} / \mathrm{O}$ ratio for our $\mathrm{mGO}$ is $\sim 5.1$ with an oxygen content of $\sim 16 \%$, confirming the above Raman and TGA results, both of which indicate a moderate degree of oxidation. It should be noted that the small $\mathrm{S}$ content seen in both GO and mGO is consistent with published data, where the effect has been attributed to covalently bonded sulphates or absorbed sulphuric acid [55]. In comparison, $\mathrm{rGO}$ typically shows $\mathrm{C} / \mathrm{O}$ values of $\sim 7.5$ [38] but can reach up to $\sim 12$ [54]. Furthermore, the oxygen content for GNP is usually around 7\% [56] or
Figure 4 a XPS survey plots on precursor graphite (black), GO obtained by $4 \mathrm{~h}$ oxidation duration (blue), and $\mathrm{mGO}$ (red), $\mathbf{b}$ deconvolution of $\mathrm{C} 1 \mathrm{~s}$ XPS spectra.
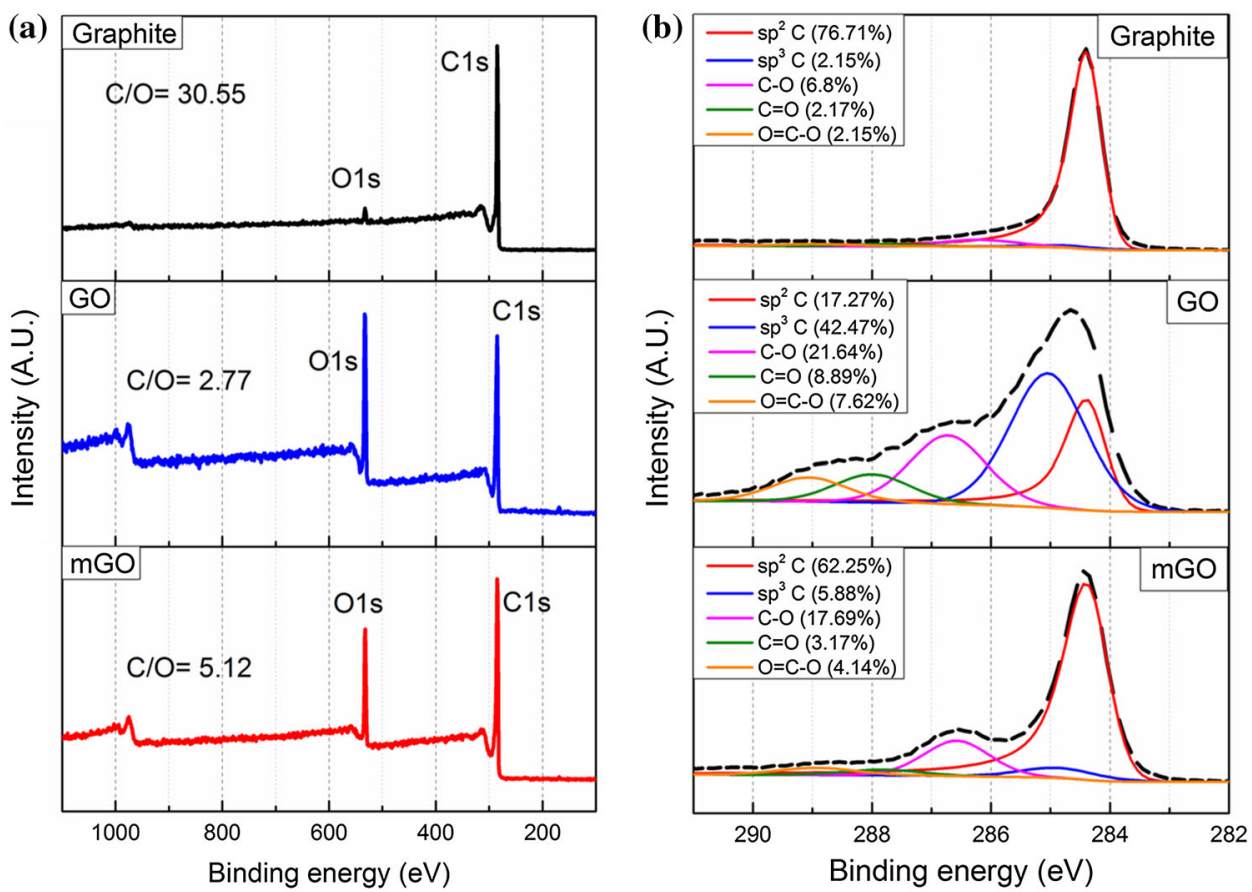
lower [57]. The electrochemical exfoliation approach mentioned earlier has shown, under specific circumstances, similar oxygen contents to $\mathrm{mGO}$, albeit with more complex production [39].

The deconvolution of the C1s core-level spectra (Fig. 4 b) shows 5 different peaks located at: $284.4 \mathrm{eV}$ $\left(s p^{2}\right.$ hybridized carbon), $285.04 \mathrm{eV}\left(s p^{3}\right.$ hybridized carbon), $286.72 \mathrm{eV}$ ( $\mathrm{C}-\mathrm{O}$ bonds from hydroxyls or epoxides), $288 \mathrm{eV}$ ( $\mathrm{C}=\mathrm{O}$ bonds from carbonyls) and $289.06 \mathrm{eV}(\mathrm{O}=\mathrm{C}-\mathrm{O}$ bonds from carboxyls). As expected, the GO shows a significant amount of $s p^{3}$ hybridized carbon atoms compared to the intact $s p^{2}$ ones, which is a result of severe oxidation by $\mathrm{KMnO}_{4}$. Furthermore, the pronounced presence of epoxides and hydroxyls, as discussed earlier, can react with the epoxy matrix [29]. The relatively increased carbonyl and carboxyl presence is also a typical result of the permanganate method [20]. Conversely, mGO shows a significantly reduced extent of $s p^{3}$ carbon hybridization and a decreased amount of functional groups compared to the GO. It is noteworthy that the dominant functionalities present on the mGO surface are epoxide and hydroxyl groups, with the presence of carbonyls and carboxyls being weaker compared to the GO but stronger compared to the graphite. Overall, the chromic method resembles the chlorate method in terms of functionalities present [20], but in a much lower amount, leading to the moderately oxidized graphene.

\section{Structure and properties of epoxy/mGO nanocomposites}

The evidence obtained above by Raman spectroscopy, TGA and XPS presents a consistent picture, whereby we conclude that the use of our chromiumbased oxidant has indeed led to the production of oxidized graphite (termed mGO here) that is characterized by reduced disorder and a reduced level of oxidation, compared with GO produced using the widely used approach first reported by Tour in 2010 . The impact of this on the structure and physical properties of a range of $\mathrm{mGO} /$ epoxy composite systems will now be described.

SEM images of fracture surfaces of the sample containing $0.5 \mathrm{vol} \%$ of mGO can be seen in Fig. $5 \mathrm{a}-\mathrm{c}$, from which a fine dispersion within the epoxy matrix is revealed. Image analysis undertaken for 74 individual flakes (Fig. 5d) shows a size distribution between 0.75 and $1.1 \mu \mathrm{m}$ for $45 \%$ of them, while $23 \%$ of the flakes exhibit a lateral size between 1.3 and $1.7 \mu \mathrm{m}$. Figure 5e, f illustrates TEM images of isolated tactoids found in the $1 \mathrm{vol} \% \mathrm{mGO}$-filled sample, with maximum lateral sizes $\sim 1.6 \mu \mathrm{m}$ and $\sim 0.8 \mu \mathrm{m}$, respectively, aligning with the abovementioned size distribution.

Typical TEM images showing the range of structural states of the mGO when dispersed at $2 \mathrm{vol} \%$ within the epoxy matrix are presented in Fig. 6. A satisfactory dispersion of the mGO forming a graphitic network within the matrix is shown. From the variation in contrast from image to image, it is evident that some areas are characterized by well-exfoliated structures (red arrows) while, in others, the mGO retains a graphitic multilayered configuration (red circle). This conforms to the Raman spectra presented above, which implies that some degree of stacking order has been preserved due to the weak oxidation. Analysis of tactoid dimensions reveals a thickness distribution with the highest probability $(>40 \%$ ) between $5 \mathrm{~nm}$ and $6 \mathrm{~nm}$. Assuming a monolayer thickness of $\sim 0.8 \mathrm{~nm}$ [6] for oxidized graphite, the majority of the examined structures contain less than 10 layers. The aggregated structures, such as that circled in red in Fig. 6, typically appeared to be $\sim 60 \mathrm{~nm}$ in thickness.

Figure 7 presents the effect of filler content on the $T_{\mathrm{g}}$ of all the nanocomposite samples considered here. In this, both weight fraction $\left(W_{\mathrm{f}}\right)$ and volume fraction $\left(V_{\mathrm{f}}\right)$ values are presented, the latter being derived from the former using Eq. 1 [19]:

$$
V_{\mathrm{f}}=\frac{W_{\mathrm{f}} \rho_{\mathrm{m}}}{W_{\mathrm{f}} \rho_{\mathrm{m}}+\left(1-W_{\mathrm{f}}\right) \rho_{\mathrm{f}}}
$$

where $\rho_{\mathrm{m}}$ is the matrix density $\left(1.16 \mathrm{~g} / \mathrm{cm}^{3}\right)$ and $\rho_{\mathrm{f}}$ is the filler density $\left(2.2 \mathrm{~g} / \mathrm{cm}^{3}[12,19]\right)$.

From the above, it can be seen that the glass transition temperature of the epoxy matrix is not detrimentally affected by the inclusion of $\mathrm{mGO}$, albeit that a statistically significant increase in $T_{\mathrm{g}}$ is observed from 4 to $12.5 \mathrm{wt} \%$. Increased $T_{\mathrm{g}}$ values have previously been reported at small GO filler contents $(>0.5$ $\mathrm{wt} \%)$ and have been ascribed either to strong fillermatrix interactions [30,34] or to the presence of wrinkled GO morphology [33], both of which serve to constrain matrix chain mobility. Here, comparable effects are seen at higher filler contents, compared to the abovementioned studies. Ultimately, at the highest filler content considered here, the $T_{\mathrm{g}}$ value drops, which has been observed before, albeit at 

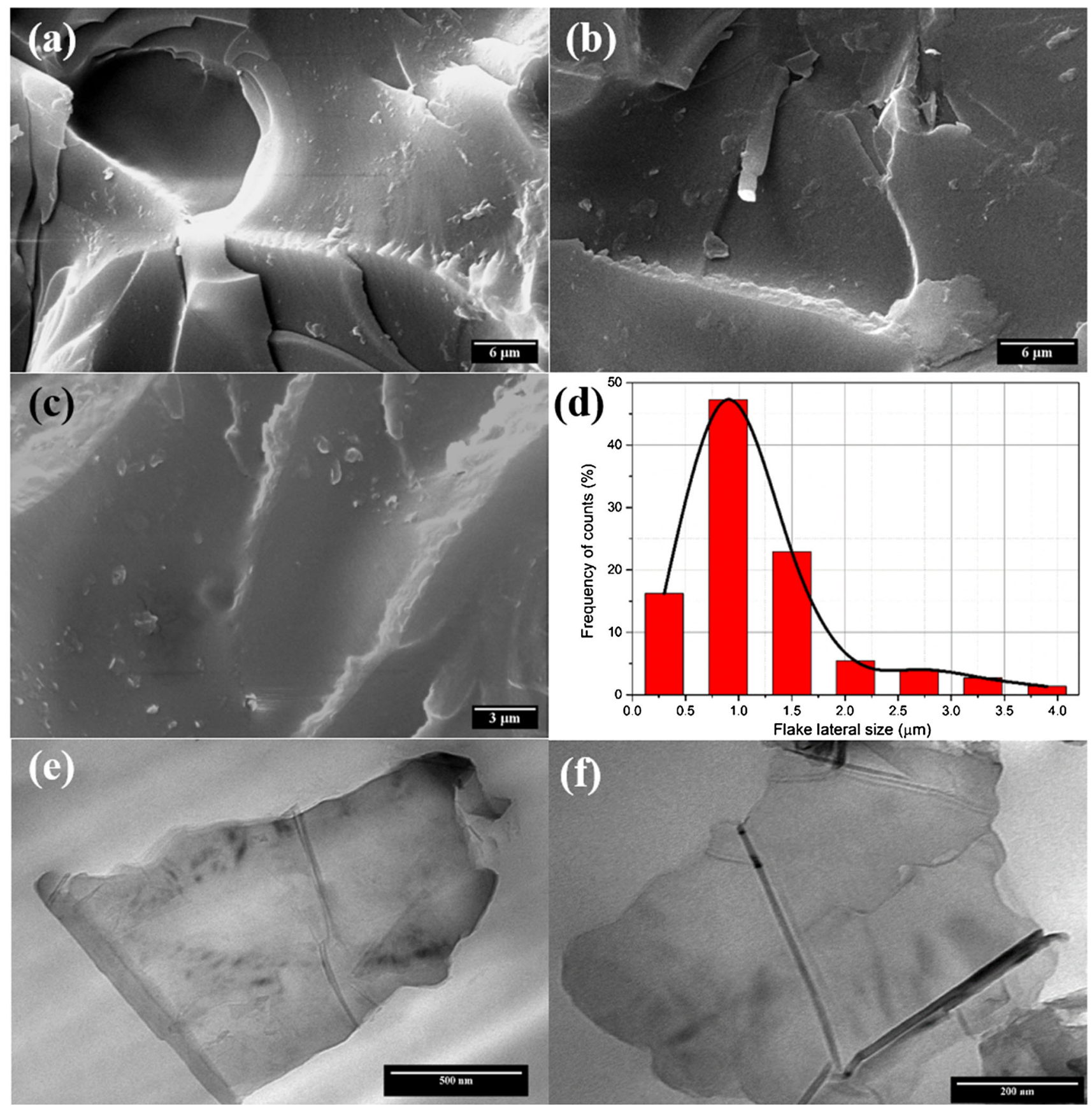

Figure 5 a-c Representative SEM images taken from the 0.53 vol\% mGO-filled nanocomposite. $\mathbf{d}$ Lateral size distribution determined by analysing 64 different flakes from the SEM images. e, f TEM images of isolated mGO flakes found at the 1 vol\% mGO-filled samples.

considerably lower filler fractions and ascribed to the high concentration of reactive functional groups that influences the stoichiometry of the epoxy crosslinking reaction [29]. Considering the latter interpretation, the $T_{\mathrm{g}}$ dependence on $\mathrm{mGO}$ content presented in Fig. 7 is consistent with the previous conclusion that the density of functional groups grafted onto the mGO surface is limited compared with conventional
GO. Evidently, mGO can be incorporated into epoxy matrices at much higher loading levels than conventional GO without adversely affecting the curing reaction of the matrix polymer.

Figure 8 shows the dependence of electrical conductivity on mGO volume fraction. At the lowest loading levels (0.1-0.4 vol\%), an increase in conductivity of about two orders of magnitude progressively 


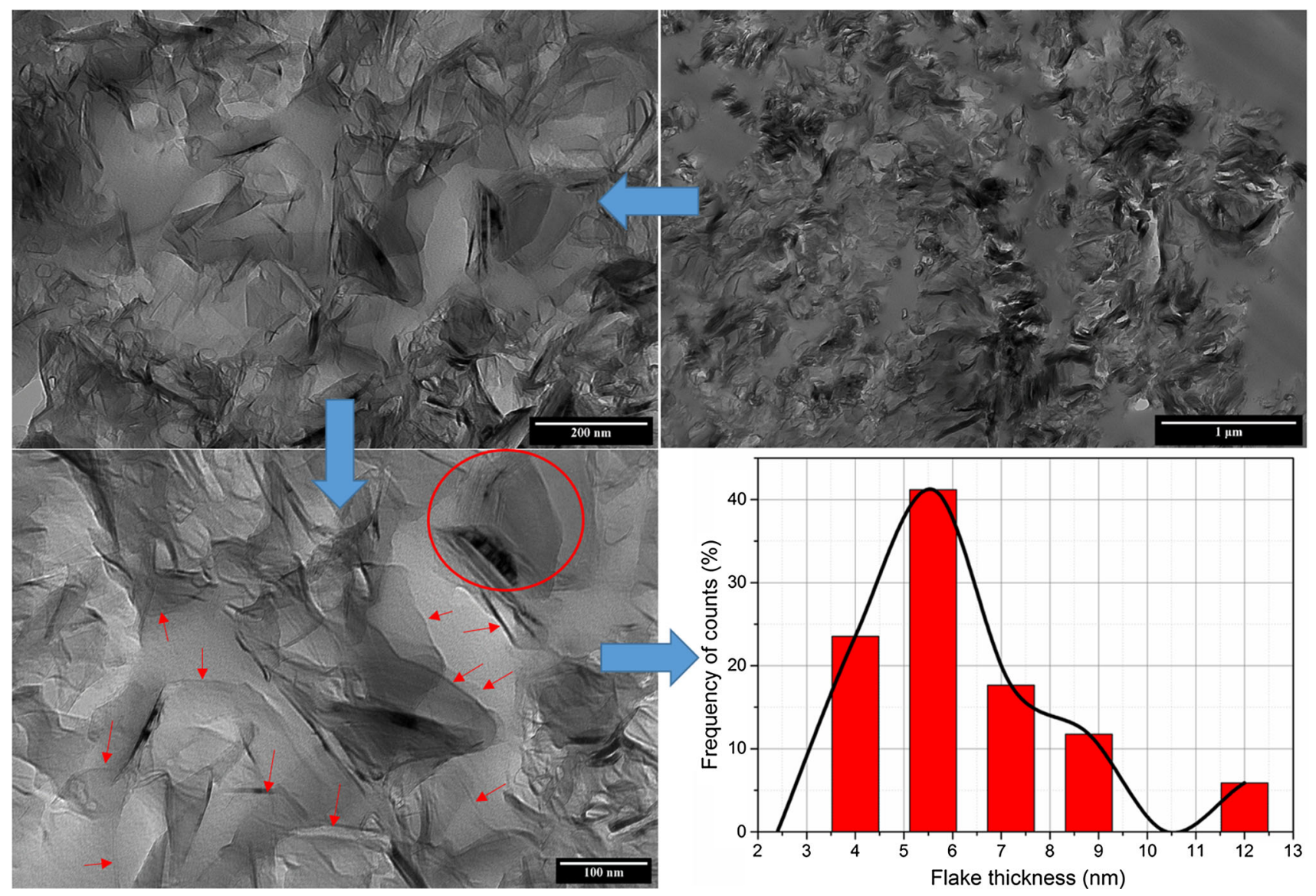

Figure 6 TEM images of the 2 vol\% mGO-filled sample at different magnification as well as thickness distribution determined by analysing 17 individual flakes.

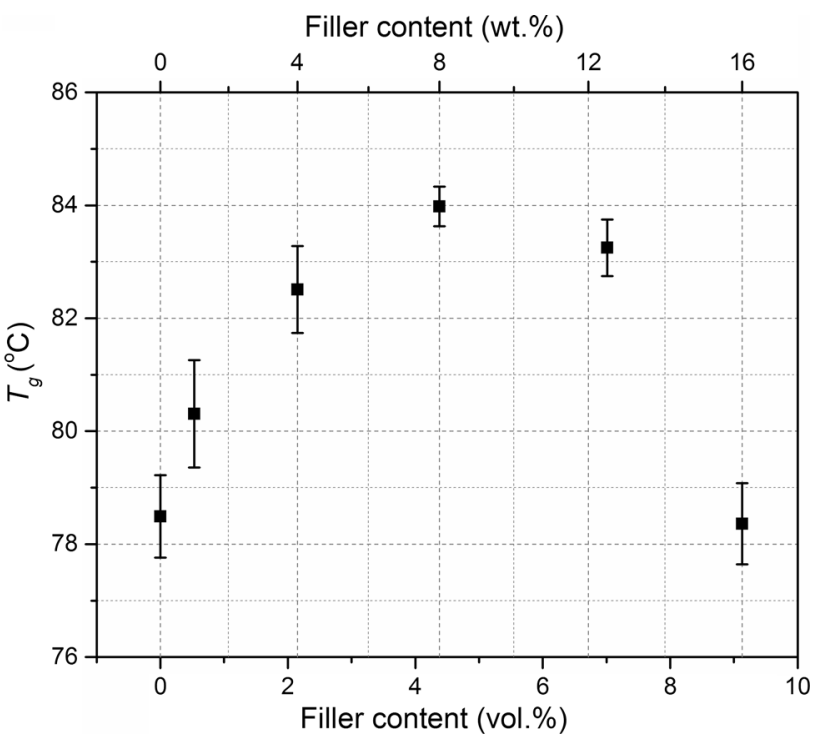

Figure 7 Weight and volume percentage of nanocomposite samples and their respective glass transition temperatures.

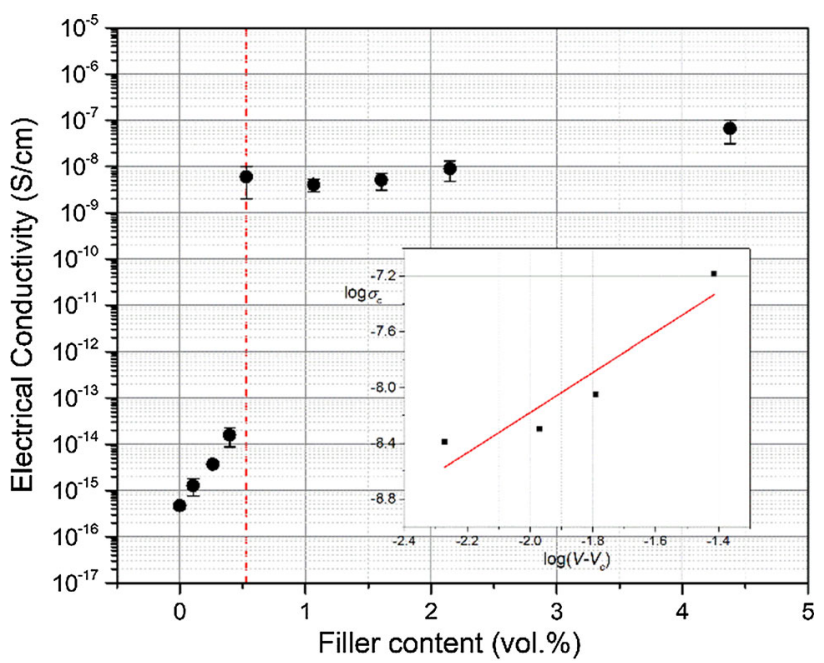

Figure 8 Variation of conductivity with mGO filler content. Percolation threshold indicated with vertical dashed red line. Inset: $\log -\log$ plot. 
occurs while, between 0.40 and 0.53 vol\%, the conductivity sharply increases by a factor approaching $10^{6}$.

In general, the conductivity of an insulating matrix containing an electrically conductive phase varies according to [58-60]:

$\sigma_{\mathrm{c}}=\sigma_{\mathrm{f}}\left(V_{\mathrm{f}}-V_{\mathrm{c}}\right)^{t}$

where $\sigma_{\mathrm{c}}$ and $\sigma_{\mathrm{f}}$ are the electrical conductivity of the composite and the filler, respectively, $t$ is a constant representing a critical exponent of the electrical conductivity and $V_{\mathrm{c}}$ is the critical fraction for which the composite exhibits a sharp conductivity increase. That is, $V_{c}$ represents the percolation threshold $\left(P_{\mathrm{t}}\right)$. Theoretically, a system containing randomly dispersed spherical, conductive particles would be expected to be characterized by a percolation threshold at about $15 \mathrm{vol} \%$ and a value of $t \approx 2 \mathrm{but}$, in practice, significant divergences occur [61]. Lower percolation thresholds are expected from fillers with high aspect ratios [62]; $t$ values can vary significantly [63-65]. Also, definitions of percolation threshold vary in the literature, with different studies variously defining this as follows: the volume fraction at which the particles form contiguous paths through physical contact, sharply increasing the electrical conductivity [66]; when the conductivity surpasses the antistatic limit $\left(10^{-8} \mathrm{~S} / \mathrm{cm}\right)$ [62]; or even when the system starts behaving nonlinearly [61]. Since the testing voltage used in this study was chosen to be low and also because the investigated systems do not display metallic behaviour, we take the percolation threshold to be determined by the first definition given above. By linearly fitting Eq. 2 to the obtained results (log$\log$ plot inset in Fig. 8), with a fixed parameter of $V_{\mathrm{c}}=0.0053$, the other two parameters $\sigma_{\mathrm{f}}$ and $t$ were calculated $\left.10^{-(5.29} \pm 0.72\right) \mathrm{S} / \mathrm{cm}$ and $1.44 \pm 0.38$, respectively (Table 2).

Comparison of these results with published data reveals that the exponential factor $t$ derived from the mGO-based systems considered here is in reasonable agreement with reported values [65], while the

Table 2 Conductivity parameters, as calculated from the percolation behaviour of mGO-filled epoxy nanocomposites

\begin{tabular}{lll}
\hline$\sigma_{\mathrm{f}}(\mathrm{S} / \mathrm{cm})$ & $V_{\mathrm{c}}$ & $t$ \\
\hline $10^{-(5.29 \pm 0.72)}$ & 0.0053 & $1.44 \pm 0.38$ \\
\hline
\end{tabular}

calculated conductivity of the mGO $\left(\sigma_{\mathrm{f}}\right)$ is, unsurprisingly, low compared to pure graphite [67] but is in reasonable agreement when compared to values derived from systems based on GO [12, 68, 69].

\section{Discussion}

In considering the significance of the results presented above, we will contrast these with the behaviour of epoxy-based systems based upon conventionally synthesized GO, such materials after reduction (rGO), after functionalization (fGO) and, finally, epoxy/graphite composites. Such systems differ from one another in a number of significant ways: surface chemistry/filler matrix interactions/filler dispersion; intrinsic electrical conductivity; influence of the included filler on the epoxy curing reaction and consequent matrix properties; complexity of filler synthesis procedure and consequent yield; ease of composite processing.

\section{Epoxy/GO nanocomposites}

The direct usage of conventional GO as a means of tailoring the electrical conductivity of epoxy-based nanocomposites has, to our knowledge, only previously been considered in the following four studies. Mancinelli et al. [70] achieved an increase of almost 2 orders of magnitude (filler content up to $0.5 \mathrm{wt} \%$ ) only by thermally treating the nanocomposite partially to reduce the GO; before thermal treatment no increase in conductivity was seen, compared with the unfilled epoxy matrix. Similar results were presented in our previous study [29] with the usage of GO in the same epoxy formulation as in the present study. Even though the filler content was extended up to 2 $\mathrm{wt} \%$, the electrical conductivity of the respective nanocomposite was the same (slightly reduced) compared to the neat epoxy, while further GO addition reduced the epoxy's $T_{\mathrm{g}}$ below ambient temperature. Kim et al. [71] did not achieve any significant conductivity increase with the usage of up to $3 \mathrm{wt} \%$ GO. Only the study presented by Tang et al. [72] achieved a modest conductivity enhancement (up to $\sim 10^{-10} \mathrm{~S} / \mathrm{cm}$ ) with a percolation threshold of 6 $\mathrm{vol} \%$. It is of considerable significance to note that the GO contents mentioned above have been reported [27-29] to affect the epoxy curing reactions as mentioned earlier. A summary of the abovementioned 
Table 3 Electrical conductivity parameters related to Epoxy/GO nanocomposites found in the literature

\begin{tabular}{|c|c|c|c|}
\hline$P_{\mathrm{t}}$ & $\sigma_{\max }(\mathrm{S} / \mathrm{cm})$ & Comments & References \\
\hline- & - & $\begin{array}{l}\text { Filler content up to } 2 \mathrm{wt} \% \\
T_{\mathrm{g}} \text { dropped below ambient temperature at higher filler contents } \\
\sigma_{\mathrm{c}} / \sigma_{\mathrm{m}} \sim 0.83\end{array}$ & {$[29]$} \\
\hline- & - & $\begin{array}{l}\text { Filler content up to } 0.5 \mathrm{wt} \% \\
\sigma_{\mathrm{c}} / \sigma_{\mathrm{m}} \sim 2.4\end{array}$ & {$[70]$} \\
\hline- & - & $\begin{array}{l}\text { Filler content up to } 3 \mathrm{wt} \% \\
\sigma_{\mathrm{c}} / \sigma_{\mathrm{m}} \sim 2.2\end{array}$ & [71] \\
\hline $6 \mathrm{vol} \%$ & $\sim 10^{-10}$ & At the same filler contents of GO our epoxy was already rubbery & {$[72]$} \\
\hline
\end{tabular}

review is presented in Table 3. Since in most cases the achieved conductivity values were relatively low, the respective data are presented as a $\sigma_{\mathrm{c}} / \sigma_{\mathrm{m}}$ ratio, where $\sigma_{\mathrm{m}}$ is the electrical conductivity of the unfilled matrix.

Thus, previous studies of systems based upon GO in epoxy indicate, at best, percolation onsets at high volume fractions, relatively small improvements in electrical conductivity and adversely perturbed matrix curing. In contrast, the mGO system developed in our work resulted in significantly lower percolation threshold (around 12 times smaller filler in terms of vol\%), higher achieved electrical conductivity and had no adverse effects on matrix curing.

\section{Epoxy/rGO and epoxy/fGO nanocomposites}

In view of the ineffective nature of GO as a means of increasing electrical conduction through epoxy-based materials, alternative strategies with this aim have used more convoluted multistep synthesis procedures to address the limitations of using GO directly. For example, Thachil et al. [32] used microwave exposure to reduce GO and, consequently, achieved a percolation threshold of $0.27 \mathrm{vol} \%$; the maximum conductivity value reported $\left(\sigma_{\max }\right)$ was, however, relatively low $\left(10^{-10} \mathrm{~S} / \mathrm{cm}\right)$. A similar percolation threshold was achieved by Hsiao et al. [73], who employed thermal reduction of $\mathrm{GO}$ at $700{ }^{\circ} \mathrm{C}$ in an argon atmosphere; in this study the $\sigma_{\max }$ reached $10^{-8} \mathrm{~S} / \mathrm{cm}$. Liang et al. [53] utilized an extensive, two-step, GO reduction process involving an initial chemical reaction with hydrazine hydrate, followed by subsequent annealing at $250{ }^{\circ} \mathrm{C}$ under $\mathrm{N}_{2}$. The resultant composite systems exhibited an $\sigma_{\max }$ of $10^{-1}$ $\mathrm{S} / \mathrm{cm}$ and a percolation threshold of $0.52 \mathrm{vol} \%$.

In the case of functionalized GO, Bao et al. [36] utilized a two-step chemical process to attach glycidol groups to the GO surface and, in this way, increased the electrical conductivity to $10^{-11} \mathrm{~S} / \mathrm{cm}$ with a percolation of $2 \mathrm{wt} \%$. Tang et al. [69, 72] achieved a much greater increase in conductivity (up to $\sim 10^{-2} \mathrm{~S} / \mathrm{cm}$ ) with similar, albeit gradual percolation onset, through surface functionalization of GO with polyetheramines. A summary of the abovementioned review is presented in Table 4.

Comparing the above results in their entirety with the behaviour of the epoxy/mGO systems reported here, it is evident that many studies report comparable behaviour with that described here while, in two cases, further processing of GO has led to systems with high bulk conductivity values (0.01-0.1 $\mathrm{S} / \mathrm{cm}$ ). Also, in all of these cases, the $\mathrm{rGO} / \mathrm{fGO}$ was introduced into the epoxy resin in a solvent, the removal of which may introduce limitations on industrial implementation [34].

\section{Epoxy/graphite-based composites}

Finally, it is worth considering the published literature concerning epoxy matrices including EG and GNP, despite the rather different chemistries and processing methodologies involved. Many studies have considered systems based upon EG, which is prepared by acidic intercalation followed by thermal expansion and then extensive sonication and solvent mixing with epoxy. Such work has reported percolation thresholds of $3 \mathrm{wt} \%$ [74], $1.3 \mathrm{vol} \%$ [75], 0.5 vol\% [76], and $1 \mathrm{phr}$ [77]. The $\sigma_{\max }$ values reported in these studies were, respectively: $10^{-3} \mathrm{~S} / \mathrm{cm} ; 10^{-5}$ $\mathrm{S} / \mathrm{cm} ; 10^{-6} \mathrm{~S} / \mathrm{cm} ; 10^{-3} \mathrm{~S} / \mathrm{cm}$. Elsewhere, Li et al. [67] used an additional $\mathrm{UV} / \mathrm{O}_{3}$ surface treatment procedure and achieved a $\sigma_{\max }$ of $10^{-4} \mathrm{~S} / \mathrm{cm}$, albeit with gradual percolation. Attempts to disaggregate graphite directly through prolonged sonication in solvent, whilst leading to systems that exhibit high 
Table 4 Electrical conductivity parameters related to epoxy/rGO and epoxy/fGO nanocomposites found in the literature

\begin{tabular}{lllll}
\hline Filler & $P_{\mathrm{t}}$ & $\sigma_{\max }(\mathrm{S} / \mathrm{cm})$ & Comments & References \\
\hline rGO & $0.27 \mathrm{vol} \%$ & $\sim 10^{-10}$ & Microwave treatment & {$[32]$} \\
& $\sim 0.25 \mathrm{wt} \%$ & $\sim 10^{-8}$ & Thermal treatment; argon atmosphere & {$[73]$} \\
& $0.52 \mathrm{vol} \%$ & $\sim 10^{-1}$ & Chemical reduction followed by thermal treatment & {$[53]$} \\
fGO & $2 \mathrm{wt} \%$ & $\sim 10^{-11}$ & Glycidol groups attached on the GO surface & {$[36]$} \\
& $\sim 2 \mathrm{vol} \%$ & $\sim 10^{-2}$ & Amine functionalization & {$[69]$} \\
& & Rubbery epoxy & \\
& $\sim 2 \mathrm{vol} \%$ & $\sim 10^{-2}$ & Gradual Percolation & Amine Functionalization \\
& & Gradual percolation &
\end{tabular}

conductivity values approaching $10^{-2} \mathrm{~S} / \mathrm{cm}$, were shown to be characterized by percolation thresholds $>8 \mathrm{wt} \%$ [78], a result we associate with low compatibility with the solvent, limited levels of disaggregation and poor dispersion.

In the case of studies of GNP/epoxy systems, direct dispersion within a solvent prior to introduction of the GNP into the resin has been reported to result in a percolation threshold $>1 \mathrm{wt} \%$ and an $\sigma_{\max }$ of $10^{-6} \mathrm{~S} / \mathrm{cm}$ [79]. More complex dispersion strategies have, however, been described, including threeroll milling [80], encapsulation with poly(vinyl pyrrolidone) combined with freeze-drying [81], spin coating (up to 12,000 rpm) [82] and AC-field induced alignment [56]; the resulting data vary widely, with $\sigma_{\max }$ values ranging from $10^{-9} \mathrm{~S} / \mathrm{cm}$ to $10^{-4} \mathrm{~S} / \mathrm{cm}$. A summary of the abovementioned review is presented in Table 5.

Table 5 Electrical conductivity parameters related to epoxy/EG and epoxy/GNP nanocomposites found in the literature

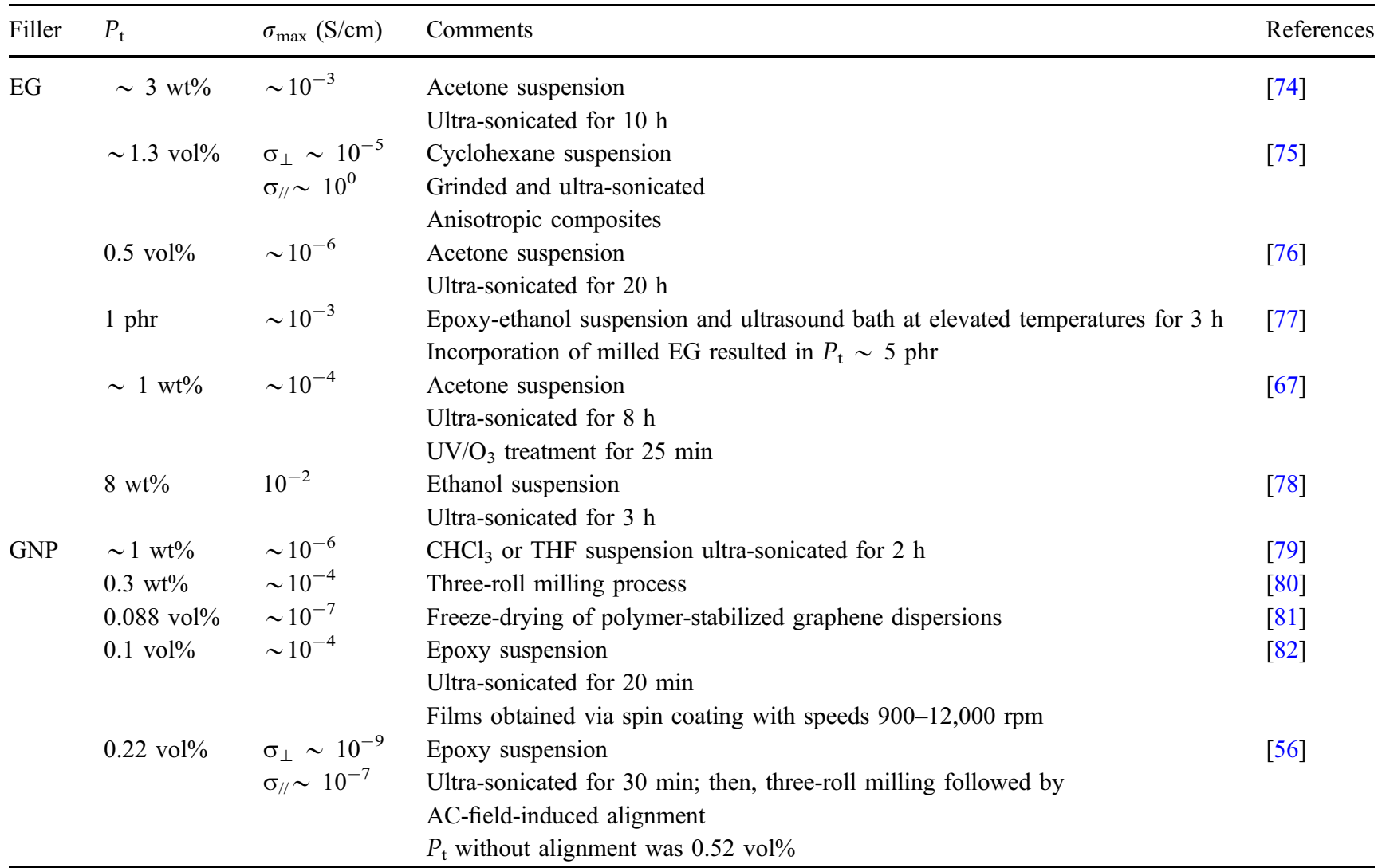


Therefore, the usage of mGO as an epoxy filler offers similar or smaller percolation thresholds than EG or GNP. However, the production of EG and GNP includes more than one step and is generally characterized by reduced yields, compared with mGO, which was synthesized with a one-step process and approaching $100 \%$ of the starting material being recovered and used, without any fractionation, in preparing the final nanocomposites.

\section{Conclusions}

Moderate oxidation of graphite was successfully achieved utilizing $\mathrm{CrO}_{3}$ instead of $\mathrm{KMnO}_{4}$ as the oxidizing agent, in a reaction medium of $\mathrm{H}_{2} \mathrm{SO}_{4}$. Raman spectroscopy, TGA and XPS demonstrated that the $\mathrm{CrO}_{3} / \mathrm{H}_{2} \mathrm{SO}_{4}$ solution mildly oxidizes the graphite, compared to previous synthetic routes. Epoxy-based nanocomposites were produced via a solvent-free method, using mGO directly produced by the above single-step synthesis method, without any subsequent chemical processing or fractionation to remove large agglomerates or other unwanted components. SEM and TEM images revealed good dispersion, suggesting that the mGO contains sufficient oxygen-based functional groups to render it compatible with the epoxy matrix. DSC determination of the influence of the mGO on the glass transition temperature of the matrix polymer revealed no detrimental effects, which indicates that the concentration of reactive groups introduced into the system as a consequence of adding the mGO was not sufficient materially to alter the epoxy curing reaction stoichiometry. The electrical conductivity measured just beyond the percolation threshold (0.53 vol\%) was $\sim 10^{-8} \mathrm{~S} / \mathrm{cm}$, an increase of some eight order of magnitude compared with the unfilled resin. Significantly, this increase surpasses the antistatic limit, a result that, to our knowledge, has not thus far been achieved with conventional GO. Indeed, comparison of the behaviour of $\mathrm{mGO}$ with a range of other graphite-based filler systems reveals an attractive combination of properties, particularly when ease of synthesis, yield and processing convenience are taken into account. As such, we believe that scope exists for future utilization or further modification.

\section{Acknowledgements}

This work was supported by the European Union's Horizon 2020 research and innovation programme under the Marie Sklodowska-Curie grant agreement [No 642771]. The XPS data were collected by Dr. Marc Walker at the Warwick Photoemission Facility, University of Warwick. The authors would also like to thank E. Senis for the SEM images.

\section{Compliance with ethical standards}

Conflict of interest The authors declare that no conflicts of interest exist.

Open Access This article is distributed under the terms of the Creative Commons Attribution 4.0 International License (http://creativecommons.org/ licenses/by/4.0/), which permits unrestricted use, distribution, and reproduction in any medium, provided you give appropriate credit to the original author(s) and the source, provide a link to the Creative Commons license, and indicate if changes were made.

\section{References}

[1] Sengupta R, Bhattacharya M, Bandyopadhyay S, Bhowmick AK (2011) A review on the mechanical and electrical properties of graphite and modified graphite reinforced polymer composites. Prog Polym Sci 36:638-670. https:// doi.org/10.1016/j.progpolymsci.2010.11.003

[2] Celzard A, Marêché JF, Furdin G (2002) Surface area of compressed expanded graphite. Carbon N Y 40:2713-2718. https://doi.org/10.1016/S0008-6223(02)00183-5

[3] Novoselov KS, Geim AK, Morozov SV et al (2004) Electric field in atomically thin carbon films. Science 306:666-669. https://doi.org/10.1126/science.1102896

[4] Geim A, Novoselov K (2007) The rise of graphene. Nat Mater 6:183-191. https://doi.org/10.1038/nmat1849

[5] Geim AK (2009) Graphene: status and prospects. Science 324:1530-1534

[6] Potts JR, Dreyer DR, Bielawski CW, Ruoff RS (2011) Graphene-based polymer nanocomposites. Polymer (Guildf) 52:5-25. https://doi.org/10.1016/j.polymer.2010.11.042

[7] Young RJ, Kinloch IA, Gong L, Novoselov KS (2012) The mechanics of graphene nanocomposites: a review. Compos Sci Technol 72:1459-1476. https://doi.org/10.1016/j.compsc itech.2012.05.005 
[8] Jang BZ, Zhamu A (2008) Processing of nanographene platelets (NGPs) and NGP nanocomposites: a review. J Mater Sci 43:5092-5101. https://doi.org/10.1007/s10853-008-2755-2

[9] Dreyer DR, Todd AD, Bielawski CW (2014) Harnessing the chemistry of graphene oxide. Chem Soc Rev 43:5288-5301. https://doi.org/10.1039/c4cs00060a

[10] Dikin DA, Stankovich S, Zimney EJ et al (2007) Preparation and characterization of graphene oxide paper. Nature 448:457-460. https://doi.org/10.1038/nature06016

[11] Lerf A, He H, Forster M, Klinowski J (1998) Structure of graphite oxide revisited. J Phys Chem B 102:4477-4482. https://doi.org/10.1021/jp9731821

[12] Stankovich S, Dikin DA, Piner RD et al (2007) Synthesis of graphene-based nanosheets via chemical reduction of exfoliated graphite oxide. Carbon N Y 45:1558-1565. https://doi. org/10.1016/j.carbon.2007.02.034

[13] Miller SG, Heimann PJ, Barlow JP, Allred RE (2007) Physical properties of exfoliated graphite nanocomposites by variation of graphite surface functionality. In: 52nd international SAMPE symposium and exhibition. Baltimore, MD

[14] Mu X, Wu X, Zhang T et al (2014) Thermal transport in graphene oxide-from ballistic extreme to amorphous limit. Sci Rep. https://doi.org/10.1038/srep03909

[15] Park S, Ruoff RS (2009) Chemical methods for the production of graphenes. Nat Nanotechnol 4:217-224. https:// doi.org/10.1038/nnano.2009.58

[16] Paredes JI, Villar-Rodil S, Martínez-Alonso A, Tascón JMD (2008) Graphene oxide dispersions in organic solvents. Langmuir 24:10560-10564. https://doi.org/10.1021/la801744a

[17] Bhattacharya M (2016) Polymer nanocomposites - a comparison between carbon nanotubes, graphene, and clay as nanofillers. Materials (Basel) 9:1-35

[18] Li Z, Young RJ, Wang R et al (2013) The role of functional groups on graphene oxide in epoxy nanocomposites. Polymer (Guildf) 54:5821-5829. https://doi.org/10.1016/j.poly mer.2013.08.026

[19] Li Z, Chu J, Yang C et al (2018) Effect of functional groups on the agglomeration of graphene in nanocomposites. Compos Sci Technol 163:116-122. https://doi.org/10.1016/ j.compscitech.2018.05.016

[20] Chua CK, Sofer Z, Pumera M (2012) Graphite oxides: effects of permanganate and chlorate oxidants on the oxygen composition. Chem A Eur J 18:13453-13459. https://doi. org/10.1002/chem.201202320

[21] Brodie BC (1859) On the atomic weight of graphite. Philos Trans R Soc Lond 149:249-259. https://doi.org/10.1098/rstl. 1859.0013

[22] Staudenmaier L (1898) Verfahren zur darstellung der graphitsäure. Berichte der Dtsch Chem Gesellschaft 31:1481-1487. https://doi.org/10.1002/cber.18980310237
[23] Hofmann U, König E (1937) Untersuchungen über Graphitoxyd. Zeitschrift für Anorg und Allg Chemie 234:311-336. https://doi.org/10.1002/zaac.19372340405

[24] Hummers WS, Offeman RE (1958) Preparation of graphitic oxide. J Am Chem Soc 80:1339

[25] Chen J, Yao B, Li C, Shi G (2013) An improved Hummers method for eco-friendly synthesis of graphene oxide. Carbon N Y 64:225-229. https://doi.org/10.1016/j.carbon.2013.07. 055

[26] Marcano DC, Kosynkin DV, Berlin JM et al (2010) Improved synthesis of graphene oxide. ACS Nano 4:4806-4814. https://doi.org/10.1021/nn1006368

[27] Galpaya D, Wang M, Yan C, et al (2013) Fabrication and characterisation of graphene oxide-epoxy nanocomposite. In: Fourth international conference on smart materials and nanotechnology in engineering, p 8793

[28] Zhou T, Nagao S, Sugahara T et al (2015) Facile identification of the critical content of multi-layer graphene oxide for epoxy composite with optimal thermal properties. RSC Adv 5:20376-20385. https://doi.org/10.1039/c4ra15881d

[29] Vryonis O, Virtanen STH, Andritsch T et al (2018) Understanding the cross-linking reactions in highly oxidized graphene/epoxy nanocomposite systems. J Mater Sci. https:// doi.org/10.1007/s10853-018-3076-8

[30] Wang X, Jin J, Song M (2013) An investigation of the mechanism of graphene toughening epoxy. Carbon N Y 65:324-333. https://doi.org/10.1016/j.carbon.2013.08.032

[31] Hsu CH, Hsu MH, Chang KC et al (2014) Physical study of room-temperature-cured epoxy/thermally reduced graphene oxides with various contents of oxygen-containing groups. Polym Int 63:1765-1770. https://doi.org/10.1002/pi.4763

[32] Sharmila TKB, Nair AB, Abraham BT et al (2014) Microwave exfoliated reduced graphene oxide epoxy nanocomposites for high performance applications. Polymer (Guildf) 55:3614-3627. https://doi.org/10.1016/j.polymer.2014.05. 032

[33] Wan Y, Tang L, Gong L et al (2013) Grafting of epoxy chains onto graphene oxide for epoxy composites with improved mechanical and thermal properties. Carbon N Y 69:467-480. https://doi.org/10.1016/j.carbon.2013.12.050

[34] Park YT, Qian Y, Chan C et al (2015) Epoxy toughening with low graphene loading. Adv Funct Mater 25:575-585. https://doi.org/10.1002/adfm.201402553

[35] Silva LCO, Silva GG, Ajayan PM, Soares BG (2015) Longterm behavior of epoxy/graphene-based composites determined by dynamic mechanical analysis. J Mater Sci 50:6407-6419. https://doi.org/10.1007/s10853-015-9193-8

[36] Bao C, Guo Y, Song L et al (2011) In situ preparation of functionalized graphene oxide/epoxy nanocomposites with 
effective reinforcements. J Mater Chem 21:13290-13298. https://doi.org/10.1039/c1jm11434d

[37] Tang X, Zhou Y, Peng M (2016) Green preparation of epoxy/graphene oxide nanocomposites using a glycidylamine epoxy resin as the surface modifier and phase transfer agent of graphene oxide. ACS Appl Mater Interfaces 8:1854-1866. https://doi.org/10.1021/acsami.5b09830

[38] Dutta D, Ganda ANF, Chih JK et al (2018) Revisiting graphene-polymer nanocomposite for enhancing anticorrosion performance: a new insight into interface chemistry and diffusion model. Nanoscale 10:12612-12624. https://doi.or $\mathrm{g} / 10.1039 / \mathrm{c} 8 \mathrm{nr} 03261 \mathrm{k}$

[39] Chen CH, Yang SW, Chuang MC et al (2015) Towards the continuous production of high crystallinity graphene via electrochemical exfoliation with molecular in situ encapsulation. Nanoscale 7:15362-15373. https://doi.org/10.1039/c $5 \mathrm{nr} 03669 \mathrm{k}$

[40] Su CY, Lu AY, Xu Y et al (2011) High-quality thin graphene films from fast electrochemical exfoliation. ACS Nano 5:2332-2339. https://doi.org/10.1021/nn200025p

[41] Skowroński JM, Jurewicz K (1991) Anodic oxidation of CrO3-graphite intercalation compounds in sulfuric acid. Synth Met 40:161-172. https://doi.org/10.1016/0379-6779 (91)91772-3

[42] Mittal J, Konno H, Inagaki M (1998) Synthesis of graphite intercalation compounds with $\mathrm{CrVI}$ compounds using $\mathrm{CrO}_{3}$ and $\mathrm{HCl}$ at room temperature. Synth Met 96:103-108. https://doi.org/10.1016/S0379-6779(98)00070-8

[43] Ebert LB, Huggins RA, Brauman JI (1974) The nature of the chromium trioxide intercalation in graphite. Carbon $\mathrm{N} \mathrm{Y}$ 12:199-208. https://doi.org/10.1016/0008-6223(74)90026-8

[44] Pei S, Cheng H-M (2012) The reduction of graphene oxide. Carbon N Y 50:3210-3228. https://doi.org/10.1016/j.carbon. 2011.11.010

[45] Vryonis O, Harrell TM, Andritsch T, et al (2018) Solvent mixing and its effect on epoxy resin filled with graphene oxide. In: 2nd IEEE international conference on dielectrics. IEEE, pp 1-4

[46] Menczel JD, Bair HE, Reading M, et al (2009) Differential scanning calorimetry (DSC). In: Menczel JD, Prime RB (eds) Thermal analysis of polymers: fundamentals and applications, Wiley, New York, pp 7-239. https://doi.org/10. 1002/9780470423837.ch2

[47] Esmaeili A, Entezari MH (2014) Facile and fast synthesis of graphene oxide nanosheets via bath ultrasonic irradiation. J Colloid Interface Sci 432:19-25. https://doi.org/10.1016/j. jcis.2014.06.055

[48] Tuinstra F, Koenig JL (1970) Raman spectrum of graphite. J Chem Phys 53:1126-1130. https://doi.org/10.1063/1. 1674108
[49] Venugopal G, Jung M-H, Suemitsu M, Kim S-J (2011) Fabrication of nanoscale three-dimensional graphite stackedjunctions by focused-ion-beam and observation of anomalous transport characteristics. Carbon N Y 49:2766-2772. https://doi.org/10.1016/j.carbon.2011.03.003

[50] Pimenta MA, Dresselhaus G, Dresselhaus MS et al (2007) Studying disorder in graphite-based systems by Raman spectroscopy. Phys Chem Chem Phys 9:1276-1291. https:// doi.org/10.1039/b613962k

[51] Krishnamoorthy K, Veerapandian M, Yun K, Kim SJ (2013) The chemical and structural analysis of graphene oxide with different degrees of oxidation. Carbon N Y 53:38-49. https://doi.org/10.1016/j.carbon.2012.10.013

[52] Eigler S, Dotzer C, Hirsch A et al (2012) Formation and decomposition of $\mathrm{CO}_{2}$ intercalated graphene oxide. Chem Mater 24:1276-1282. https://doi.org/10.1021/cm203223z

[53] Liang J, Wang Y, Huang Y et al (2009) Electromagnetic interference shielding of graphene/epoxy composites. Carbon N Y 47:922-925. https://doi.org/10.1016/j.carbon.2008. 12.038

[54] Papageorgiou DG, Kinloch IA, Young RJ (2017) Mechanical properties of graphene and graphene-based nanocomposites. Prog Mater Sci 90:75-127. https://doi.org/10.1016/j.pmatsci. 2017.07.004

[55] Shen L, Zhang L, Wang K et al (2018) Analysis of oxidation degree of graphite oxide and chemical structure of corresponding reduced graphite oxide by selecting different-sized original graphite. RSC Adv 8:17209-17217. https://doi.org/ 10.1039/c8ra01486h

[56] Wu S, Ladani RB, Zhang J et al (2015) Aligning multilayer graphene flakes with an external electric field to improve multifunctional properties of epoxy nanocomposites. Carbon N Y 94:607-618. https://doi.org/10.1016/j.carbon.2015.07. 026

[57] Wang Y, Yu J, Dai W et al (2015) Enhanced thermal and electrical properties of epoxy composites reinforced with graphene nanoplatelets. Polym Compos 36:556-565. http s://doi.org/10.1002/pc.22972

[58] Lux F (1993) Models proposed to explain the electrical conductivity of mixtures made of conductive and insulating materials. J Mater Sci 28:285-301. https://doi.org/10.1007/ BF00357799

[59] McLachlan DS (1986) Equations for the conductivity of macroscopic mixtures. J Phys C Solid State Phys 19:1339-1354. https://doi.org/10.1088/0022-3719/19/9/007

[60] Kirkpatrick S (1973) Percolation and conduction. Rev Mod Phys 45:574-588. https://doi.org/10.1103/RevModPhys.45. 574 
[61] Yang X, Hu J, Chen S, He J (2016) Understanding the percolation characteristics of nonlinear composite dielectrics. Sci Rep 6:1-11. https://doi.org/10.1038/srep30597

[62] Gojny FH, Wichmann MHG, Fiedler B et al (2006) Evaluation and identification of electrical and thermal conduction mechanisms in carbon nanotube/epoxy composites. Polymer (Guildf) 47:2036-2045. https://doi.org/10.1016/j.polymer.2 006.01 .029

[63] Gonon P, Boudefel A (2006) Electrical properties of epoxy/ silver nanocomposites. J Appl Phys 99:024308. https://doi. org/10.1063/1.2163978

[64] McLachlan DS, Chiteme C, Park C et al (2005) AC and DC percolative conductivity of single wall carbon nanotube polymer composites. J Polym Sci Part B Polym Phys 43:3273-3287. https://doi.org/10.1002/polb.20597

[65] Nan C-W, Shen Y, Ma J (2010) Physical properties of composites near percolation. Annu Rev Mater Res 40:131-151. https://doi.org/10.1146/annurev-matsci-070909 $-104529$

[66] Stankovich S, Dikin DA, Dommett GHB et al (2006) Graphene-based composite materials. Nature 442:282-286. https://doi.org/10.1038/nature04969

[67] Li J, Sham ML, Kim JK, Marom G (2007) Morphology and properties of UV/ozone treated graphite nanoplatelet/epoxy nanocomposites. Compos Sci Technol 67:296-305. https:// doi.org/10.1016/j.compscitech.2006.08.009

[68] Deka MJ, Baruah U, Chowdhury D (2015) Insight into electrical conductivity of graphene and functionalized graphene: role of lateral dimension of graphene sheet. Mater Chem Phys 163:236-244. https://doi.org/10.1016/j.matche mphys.2015.07.036

[69] Tang G, Jiang ZG, Li X et al (2014) Electrically conductive rubbery epoxy/diamine-functionalized graphene nanocomposites with improved mechanical properties. Compos Part B Eng 67:564-570. https://doi.org/10.1016/j.compositesb.2 014.08 .013

[70] Mancinelli P, Heid TF, Fabiani D, et al (2013) Electrical conductivity of graphene-based epoxy nanodielectrics. In: Annual report-conference on electrical insulation and dielectric phenomena, CEIDP, pp 772-775. https://doi.org/ 10.1109/CEIDP.2013.6748282

[71] Kim J, Im H, Kim J, Kim J (2012) Thermal and electrical conductivity of $\mathrm{Al}(\mathrm{OH}) 3$ covered graphene oxide nanosheet/ epoxy composites. J Mater Sci 47:1418-1426. https://doi. org/10.1007/s10853-011-5922-9

[72] Tang G, Jiang ZG, Li X et al (2014) Simultaneous functionalization and reduction of graphene oxide with polyetheramine and its electrically conductive epoxy nanocomposites. Chinese J Polym Sci (Engl Ed) 32:975-985. https://doi.org/10.1007/s10118-014-1488-8
[73] Hsiao MC, Ma CCM, Chiang JC et al (2013) Thermally conductive and electrically insulating epoxy nanocomposites with thermally reduced graphene oxide-silica hybrid nanosheets. Nanoscale 5:5863-5871. https://doi.org/10.103 9/c3nr01471a

[74] Jović N, Dudić D, Montone A et al (2008) Temperature dependence of the electrical conductivity of epoxy/expanded graphite nanosheet composites. Scr Mater 58:846-849. https://doi.org/10.1016/j.scriptamat.2007.12.041

[75] Celzard A, McRae E, Marêché JF et al (1996) Composites based on micron-sized exfoliated graphite particles: electrical conduction, critical exponents and anisotropy. J Phys Chem Solids 57:715-718. https://doi.org/10.1016/0022-369 7(95)00337-1

[76] Zheng C, Fan Z, Wei T, Luo G (2009) Temperature dependence of the conductivity behavior of graphite nanoplateletfilled epoxy resin composites. J Appl Polym Sci 113:1515-1519. https://doi.org/10.1002/app.30009

[77] Jia W, Tchoudakov R, Narkis M, Siegmann A (2005) Performance of expanded graphite and expanded milled-graphite fillers in thermosetting resins. Polym Compos 26:526-533. https://doi.org/10.1002/pc.20123

[78] Tien DH, Park J, Han SA et al (2011) Electrical and thermal conductivities of stycast 1266 epoxy/graphite composites. J Korean Phys Soc 59:2760-2764. https://doi.org/10.3938/ jkps.59.2760

[79] Monti M, Rallini M, Puglia D et al (2013) Morphology and electrical properties of graphene-epoxy nanocomposites obtained by different solvent assisted processing methods. Compos Part A Appl Sci Manuf 46:166-172. https://doi.org/ 10.1016/j.compositesa.2012.11.005

[80] Chandrasekaran S, Seidel C, Schulte K (2013) Preparation and characterization of graphite nano-platelet (GNP)/epoxy nano-composite: mechanical, electrical and thermal properties. Eur Polym J 49:3878-3888. https://doi.org/10.1016/j.e urpolymj.2013.10.008

[81] Wajid AS, Tanvir Ahmed HS, Das S et al (2013) High-performance pristine graphene/epoxy composites with enhanced mechanical and electrical properties. Macromol Mater Eng 298:339-347. https://doi.org/10.1002/mame.201200043

[82] Ghaleb ZA, Mariatti M, Ariff ZM (2014) Properties of graphene nanopowder and multi-walled carbon nanotubefilled epoxy thin-film nanocomposites for electronic applications: the effect of sonication time and filler loading. Compos Part A Appl Sci Manuf 58:77-83. https://doi.org/ 10.1016/j.compositesa.2013.12.002

Publisher's Note Springer Nature remains neutral with regard to jurisdictional claims in published maps and institutional affiliations. 